\section{Typ-1-Diabetes: Pumpen auf dem Vormarsch}

$\mathrm{B}$ ei der Insulintherapie von Kindern hat in den letzten Jahren ein Paradigmenwechsel stattgefunden. Heute wird rund die Hälfte der Typ-1Diabetiker mit Insulinpumpen versorgt. Dass deutsche Kinder mit Typ-1-Diabetes im europäischen Vergleich bei den $\mathrm{HbA}_{1 \mathrm{c}}$-Werten deutlich besser abschneiden als Kinder in anderen Ländern, könnte daran liegen, dass in Deutschland weit mehr Kinder mit einer Pumpe versorgt werden als etwa in England, vermutete Olga Kordonouri vom Diabeteszentrum für Kinder und Jugendliche in Hannover. In Deutschland würden etwa $50 \%$ der Kinder mit mit einem Pumpensystem versorgt, während dies in England lediglich bei $14 \%$ der Fall sei.
Die auf dem Markt befindlichen Pumpensysteme erlauben eine optimale Insulinversorgung der kleinen Patienten und entbinden sie von ständigen Glukosemessungen. So kommunizieren bei der Sensor-unterstützten Pumpentherapie (SuP) Pumpe und Glukosesensoren, indem die kontinuierlich ermittelten subkutanen Gewebeglukosewerte automatisch an die Pumpe gesendet werden. Damit kann nicht nur eine optimale Insulinversorgung gewährleistet werden, sondern die Insulingabe wird über die Alarmfunktion bei drohender oder eingetretener Hypoglykämie automatisch abgeschaltet, fasste Kononouri die größten Vorteile der Pumpentherapie zusammen. Hinzu kommen Dokumentation,
Bolus-Rechner, Analysen und vieles mehr, die eine weitgehend physiologische Versorgung der Kinder mit deutlicher Reduktion des Hypoglykämierisikos weiter erleichtern. Dadurch dass Diabetes „sichtbar“ wird, erhöht sich die Akzeptanz der Therapie und damit die Compliance bei Kindern und Eltern. „Bisher war die SuP keine Leistung der GKV. Von der neuen Gesetzeslage erhoffen wir uns, dass deutlich mehr Kinder als bisher von ihr profitieren können", so die Hoffnung der Expertin. Mit GBABeschluss vom 16.6.2016 sind die Pumpen-Systeme zu Lasten der GKV verordungsfähig, wenn mit einer intensivierten Insulintherapie das festgelegte individuelle Therapieziel nicht erreicht wird.

\section{Dr. Claudia-Viktoria Schwörer}

Kordonouri O. Update Diabetes

\title{
STIKO-Empfehlungen zur HPV-Impfung
}

$\mathrm{H}$ PV sind hochinfektiöse Viren. Während in der Vergangenheit gegen die besonders karzinogenen Serotypen 16 und 18 geimpft wurde, steht seit 2016 ein für Mädchen und Jungen ab 9 Jahren zugelassener neunvalenter Impfstoff zur Verfügung. Zugelassen ist der Impfstoff zur Prävention von Vorstufen maligner Läsionen und Karzinomen an Zervix, Vulva, Vagina und Anus sowie Genitalwarzen, die mit den HPVSerotypen 6, 11, 16, 18, 31, 33, 45, 52 und 58 assoziiert sind. Zugelassen ist ein 2-Dosen-Schema im Alter von 9-14 Jahren, sowie ab dem 15. Lebensjahr ein 3-Dosen-Schema (0, 2, 6 Monate).

Die STIKO empfiehlt zur Reduktion der Krankheitslast durch den Gebärmutterhalskrebs für alle Mädchen eine HPV-Impfung, wobei mit der Grundimmunisierung möglichst früh begonnen werden sollte. Für Mädchen von 9-13 Jahren $\left(\right.$ Gardasil ${ }^{\circledR}$ ) und von 9-14 Jahren (Cervarix ${ }^{\oplus}$, Gardasil $^{\oplus}$ 9) wird ein 2-Dosen-Schema zur Grundimmunisierung empfohlen; für Mädchen von 15-17 Jahren ist die Nachholimpfung mit dem 3-Dosen-Schema vorgesehen. Die Impfserie sollte spätestens zum vollendeten 18. Lebensjahr abgeschlossen sein. Opti- malen Schutz bietet die Impfung dann, wenn die Impfserie vor dem ersten Geschlechtsverkehr abgeschlossen ist.

Wie Dr. Miriam Wiese-Posselt, Mitglied des Fachgebiets Impfprävention am Robert-Koch-Institut und der AG HPV der STIKO, Berlin, berichtete, schließt sich die STIKO den Empfehlungen der Hersteller an, nach dem eine Impfserie mit dem Impfstoff beendet werden soll, mit dem sie begonnen wurde. Zudem kann eine mit dem viervalenten Impfstoff begonnene Impfserie mit dem neunvalenten beendet werden, wenn der viervalente nicht mehr zur Verfügung steht. Die Patienten müssen jedoch darüber aufgeklärt werden, dass für die fünf Serotypen 31, 33, 45, 52 und 58 kein ausreichender Schutz gewährleistet werden kann. Dr.Claudia-Viktoria Schwörer

Wiese-Posselt M. Aktueller Stand zur HPVImpfung (9-valenter HPV-Impfstoff, JungenImpfung) 